\title{
Densidad vascular/de perfusión en dos protocolos de angiotomografía de coherencia óptica ¿Intercambiables?
}

\author{
Gerardo Monares-Zepeda1, Margarita Montano², Luisa A. Bonilla², Alan Chew-Bonilla ${ }^{2}$ y \\ Virgilio Lima-Gómez ${ }^{*}$ \\ ${ }^{1}$ Servicio de Retina, Clínica de Microcirugía Ocular Avanzada Dr. Enrique Monares García, Toluca, Edo. Méx.; '2Oftalmología, Práctica privada, \\ Toluca, Edo. Méx.; ${ }^{3}$ Servicio de Oftalmología, Hospital Juárez de México, Ciudad de México, México
}

\section{Resumen}

Introducción: Distintos protocolos de angiotomografía de coherencia óptica evalúan la mácula. Objetivo: R2) entre las densidades vascular y de perfusión de dos protocolos de angiotomografía de coherencia óptica, para determinar si sus mediciones podían intercambiarse. Método: Estudio observacional, comparativo, prospectivo, transversal entre dos protocolos de angiotomografía de coherencia óptica (AngioPlex, Zeiss) en sujetos sanos. Se identificó la R2 entre las densidades vascular y de perfusión central, interna y completa (protocolo de 3 x $3 \mathrm{~mm}$ ), y central, interna, externa y completa (protocolo de $6 \times 6 \mathrm{~mm}$ ). Resultados: 78 ojos, mediana de edad 23 años. Hubo R2 altas entre las densidades interna y completa del protocolo de 3 × $3 \mathrm{~mm}$ (0.96), externa y completa del de 6 × $6 \mathrm{~mm}$ (0.96), y centrales vasculares y de perfusión ( $\geq 0.96) ;$ la $R 2$ entre las densidades centrales vascular y de perfusión de distintos protocolos fue $\leq 0.71$. Conclusiones: Las densidades vasculares y de perfusión tienen R2 alta dentro de un protocolo, pero no entre protocolos, porque estos miden preferentemente zonas distintas, lo cual limita intercambiar mediciones.

PALABRAS CLAVE: Angiografía por tomografía de coherencia óptica. Densidad de vasos. Densidad de perfusión. Mácula. Protocolo de rastrero.

\section{Vessel/ perfusion density in two optical coherence tomography angiography protocols. Interchangeable?}

\begin{abstract}
Introduction: Different optical coherence tomography angiography (OCTA) scanning protocols evaluate the macula. Objective: To compare the determination coefficients (R2) between vessel and perfusion densities of two OCTA scanning protocols, to learn whether their metrics could be interchanged. Method: Non-experimental, comparative, prospective, observational, cross-sectional study, between two OCTA scanning protocols (Angioplex, Zeiss) in healthy subjects. We found the R2 between central, inner, and full densities ( $3 \times 3 \mathrm{~mm}$ protocol), and between central, inner, outer and full densities (6x $6 \mathrm{~mm}$ protocol), both for vessel and perfusion densities. Results: 78 eyes, median age 23 years. There were high R2 between inner and full densities in the $3 \times 3 \mathrm{~mm}$ protocol (0.96), between outer and full densities in the $6 \times 6 \mathrm{~mm}$ protocol (0.96) and between central vessel and perfusion densities ( $\geq 0.96)$; $R 2$ between central vessel and perfusion densities of different protocols $(\leq 0.71)$.
\end{abstract}

${ }^{*}$ Correspondencia:

Virgilio Lima-Gómez.

E-mail: forscher7@gmail.com
Gac Med Mex. 2021;157:166-173

Disponible en PubMed

www.gacetamedicademexico.com

0016-3813/@ 2020 Academia Nacional de Medicina de México, A.C. Publicado por Permanyer. Este es un artículo open access bajo la licencia CC BY-NC-ND (http://creativecommons.org/licenses/by-nc-nd/4.0/). 
Conclusions: Vessel and perfusion densities have high determination coefficients within a scanning protocol, but not between protocols, because each preferentially measures different macular areas. The metrics of different protocols should not be interchanged for follow-up.

KEY WORDS: Optical coherence tomography angiography. Vessel density. Perfusion density. Macula. Scanning protocol.

\section{Introducción}

Las enfermedades de la mácula afectan a la región de la retina que tiene la máxima función visual; la angiografía por tomografía de coherencia óptica es un estudio diagnóstico que mide los plexos capilares de la retina y puede localizar objetivamente cambios por enfermedades vasculares en la mácula. ${ }^{1,2}$ Las mediciones cuantitativas de la angiografía por tomografía de coherencia óptica evalúan la densidad vascular parafoveal y miden la zona avascular foveal y la perfusión macular; la angiografía por tomografía de coherencia óptica Angioplex (Zeiss) genera mediciones reproducibles de estas variables mediante protocolos de rastreo de $3 \times 3 \mathrm{~mm}{ }^{4}$ y $6 \times 6 \mathrm{~mm}{ }^{5}$ y proporciona valores central, interna y completa $(3 \times 3 \mathrm{~mm})$ y valores central, interna, externa y completa $(6 \times 6 \mathrm{~mm})$. Aunque ambos protocolos miden los mismos parámetros vasculares, el coeficiente de determinación alto entre las mediciones centrales disminuye en las zonas internas, lo cual limita el seguimiento cuando la evaluación entre visitas alterna entre protocolos de $3 \times 3 \mathrm{~mm}$ y de $6 \times 6 \mathrm{~mm}$. ${ }^{6}$

Algunos estudios han comparado las mediciones de angiografía por tomografía de coherencia óptica entre distintos equipos. ${ }^{7-10}$ Aunque la descripción ha mostrado diferencias, aún resta saber si alguna de las mediciones puede intercambiarse directamente entre los protocolos de $3 \times 3 \mathrm{~mm}$ y de $6 \times 6 \mathrm{~mm}$ del Angioplex; otro tema por resolver es la contribución de la densidad vascular a la densidad de perfusión macular, porque cada evaluación requiere un rastreo distinto y el valor de un solo estudio como predictor del otro se desconoce.

La contribución de los parámetros vasculares a la perfusión macular en sujetos sanos es una referencia para identificar cambios tempranos en enfermedades vasculares retinianas. Llevamos a cabo un estudio para determinar las correlaciones y los coeficientes de determinación entre las variables vasculares y de perfusión en una muestra de adultos sanos, con los protocolos de angiografía por tomografía de coherencia óptica de $3 \times 3 \mathrm{~mm}$ y de $6 \times 6 \mathrm{~mm}$, y para determinar la probabilidad de intercambiar directamente las mediciones entre ellos.

\section{Material y método}

Se desarrolló un estudio observacional, prospectivo, comparativo y transversal en sujetos sanos de la región central de México, del 1 de enero al 30 de septiembre de 2018, para comparar la distribución y la correlación de las densidades vascular y de perfusión entre dos protocolos de rastreo del Angioplex. Todos los sujetos se reclutaron en una clínica privada de oftalmología. El estudio se apegó a los principios de la Declaración de Helsinki y se mantuvo en forma anónima la identidad de los sujetos.

Se incluyeron sujetos sanos de cualquier sexo con edad de 18 a 30 años, quienes tuvieran una agudeza visual corregida de 0.0 en logMAR y aceptaran participar en el estudio mediante consentimiento informado por escrito. Se excluyeron los ojos con anormalidades estructurales de la mácula, grosor macular anormal, una intensidad de señal de la angiografía por tomografía de coherencia óptica $<7$, o cualquier artefacto de la imagen o error de medición. Los ojos con valores estructurales mediante tomografía de coherencia óptica, o valores de la angiografía por tomografía de coherencia óptica por fuera de tres desviaciones estándar del promedio de la muestra se eliminaron.

En cada ojo se midió la agudeza visual con corrección y se examinó el fondo del ojo. En todos los sujetos se tomó una tomografía de coherencia óptica estructural y una angiografía por tomografía de coherencia óptica con el equipo Cirrus HD Angioplex (Carl Zeiss Meditec, Dublin CA) en ambos ojos. Se usaron dispositivos de alineación y seguimiento ocular para obtener un cubo de grosor macular $(512 \times 128)$ y protocolos de densidades vascular y de perfusión de 3 x $3 \mathrm{~mm}$ y de $6 \times 6 \mathrm{~mm}$. Un investigador evaluó las imágenes de grosor para descartar cambios de la retina que pudieran haberse omitido durante la oftalmoscopia habitual. La angiografía por tomografía de coherencia óptica midió las siguientes zonas de densidad vascular y de perfusión: en el protocolo de $3 \mathrm{x}$ $3 \mathrm{~mm}$ : central, interna, y completa; en el protocolo de 6 × $6 \mathrm{~mm}$ : central, interna, externa y completa. También se midió la zona avascular foveal. 
Se usó una prueba de Kolmogorov-Smirnov para determinar si las variables tenían una distribución normal. Se calcularon la mediana y el rango intercuartílico para cada variable. Los valores de densidad vascular central, densidad de perfusión central y área de la zona avascular foveal del protocolo de $3 \times 3 \mathrm{~mm}$ se compararon con los valores correspondientes del protocolo de $6 \times 6 \mathrm{~mm}$ mediante la prueba $\mathrm{t}$ de Wilcoxon, para determinar si podían intercambiarse directamente.

Se calcularon las correlaciones entre las variables correspondientes de ambos protocolos con la prueba de Spearman. Para las correlaciones por arriba de 0.9 y por debajo de -0.8 se calculó el coeficiente de determinación $\left(R^{2}\right)$ con un modelo lineal. Se consideraron significativos los valores de $p<0.05$ y de $R^{2}>0.9$.

\section{Resultados}

Se incluyeron 112 ojos y se eliminaron 44, lo cual dejó 78 ojos de 56 sujetos para el análisis; 47 ojos eran de mujeres (60\%); la edad fue de 18 a 30 años (mediana: 23, 21-25 años). La prueba de KolmogorovSmirnov detectó distribuciones anormales para la densidad vascular interna, densidad vascular completa, perfusión interna, perfusión externa y perfusión completa del protocolo de $6 \times 6 \mathrm{~mm}$.

La Tabla 1 presenta la distribución de las variables de densidad vascular, área de la zona avascular foveal y densidad de perfusión en los protocolos de $3 \times 3 \mathrm{~mm}$ y de $6 \times 6 \mathrm{~mm}$, los valores más altos de las medianas se encontraron en la densidad interna del protocolo de $3 \times 3 \mathrm{~mm}$ y en la densidad externa del protocolo de $6 \times 6 \mathrm{~mm}$; los rangos intercuartílicos de la densidad interna en los protocolos de $3 \times 3 \mathrm{~mm}$ y $6 \times 6 \mathrm{~mm}$ fueron excluyentes. Los rangos intercuartílicos de la perfusión interna en el protocolo de $3 \times 3 \mathrm{~mm}$ y en el de $6 \times 6 \mathrm{~mm}$ también fueron excluyentes.

La Tabla 2 presenta la comparación entre medianas de las variables correspondientes; la densidad vascular central y el área de la zona avascular foveal tuvieron medianas mayores en el protocolo de $3 \times 3 \mathrm{~mm}$, mientras que la mediana de la perfusión central fue mayor en el protocolo de $6 \times 6 \mathrm{~mm}$.

La Tabla 3 muestra las correlaciones relevantes; aquellas entre la edad y todas las variables de angiografía por tomografía de coherencia óptica fueron $\mathrm{Rho}=0.25$ o menores. Aunque la densidad vascular interna y la densidad vascular completa tuvieron una correlación alta en el protocolo de $3 \times 3 \mathrm{~mm}$, en el protocolo de $6 \times 6 \mathrm{~mm}$ su correlación fue menor que la existente entre la densidad vascular externa y la densidad vascular completa. Esta última correlación fue tan alta como la existente entre la densidad vascular interna y la densidad vascular completa en el protocolo de $3 \times 3 \mathrm{~mm}$.

Las correlaciones más altas se presentaron entre la densidad vascular central y la densidad de perfusión central en cada protocolo; la correlación entre la densidad vascular central y el área de la zona avascular foveal fue alta, pero no tanto como la existente entre la densidad vascular central y la densidad de perfusión central. Se encontró lo mismo entre la densidad de perfusión central y el área de la zona avascular foveal en ambos protocolos.

Los coeficientes de determinación más altos se encontraron entre las densidades centrales vascular y de perfusión en ambos estudios $\left(3 \times 3 \mathrm{~mm} \mathrm{R} \mathrm{R}^{2}=0.96\right.$, $6 \times 6 \mathrm{~mm} \mathrm{R} \mathrm{R}^{2}=0.98$ ); en el protocolo de $3 \times 3 \mathrm{~mm}$ los coeficientes de determinación entre la densidad vascular central y el área de la zona avascular foveal $\left(R^{2}=0.77\right)$ y entre la densidad de perfusión central y el área de la zona avascular foveal $\left(R^{2}=0.71\right)$ fueron discretamente mayores que los encontrados para las mismas variables en el protocolo de $6 \times 6 \mathrm{~mm}$ (densidad vascular $R^{2}=0.711$, perfusión $R^{2}=0.67$ )

El coeficiente de determinación entre la densidad vascular central en el protocolo de $3 \times 3 \mathrm{~mm}$ y la densidad de perfusión central en el protocolo de $6 \times 6 \mathrm{~mm}$ fue $\mathrm{R}^{2}=0.66$; el coeficiente de determinación entre la densidad vascular central en el protocolo de $6 \times 6 \mathrm{~mm}$ y la perfusión central en el protocolo de $3 \times 3 \mathrm{~mm}$ fue $\mathrm{R}^{2}=0.68$.

En el protocolo de $3 \times 3 \mathrm{~mm}$ el coeficiente de determinación entre la densidad vascular interna y la densidad vascular completa fue $R^{2}=0.95$, y entre densidad de perfusión interna y densidad de perfusión completa fue $\mathrm{R}^{2}=0.96$. En el protocolo de $6 \times 6$ $\mathrm{mm}$ los coeficientes de determinación fueron $R^{2}=0.76$ entre la densidad vascular interna y la densidad vascular completa, y $\mathrm{R}^{2}=0.96$ entre la densidad vascular externa y la densidad vascular completa (Figura 1); fueron $R^{2}=0.76$ entre la densidad de perfusión interna y la densidad de perfusión completa, y $\mathrm{R}^{2}=0.96$ entre la densidad de perfusión externa y la densidad de perfusión completa (Figura 2).

\section{Discusión}

Se encontró una correlación alta entre las densidades vascular y de perfusión en ambos protocolos del Angioplex ( $3 \times 3 \mathrm{~mm}$ y $6 \times 6 \mathrm{~mm}$ ); los valores de estas 
Tabla 1. Distribución de la densidad vascular $\left(\mathrm{mm}^{-1}\right)$, área de la zona avascular foveal y densidad de perfusión (\%) en los protocolos de $3 \times 3 \mathrm{~mm}$ y $6 \times 6 \mathrm{~mm}$

\begin{tabular}{|c|c|c|}
\hline Variable & Rango & $\begin{array}{c}\text { Mediana, rango } \\
\text { intercuartílico }\end{array}$ \\
\hline $\begin{array}{l}\text { Densidad vascular central } \\
3 \times 3 \mathrm{~mm}\end{array}$ & $6.3-15.4$ & $10.3,8.6-12.0$ \\
\hline $\begin{array}{l}\text { Densidad vascular interna } \\
3 \times 3 \mathrm{~mm}\end{array}$ & $19.6-24.4$ & $22.9,21.7-23.4$ \\
\hline $\begin{array}{l}\text { Densidad vascular completa } 3 \\
\text { × } 3 \mathrm{~mm}\end{array}$ & 18.3-23.0 & $21.4,20.5-22.0$ \\
\hline $\begin{array}{l}\text { Densidad vascular central } \\
6 \times 6 \mathrm{~mm}\end{array}$ & $5.3-14.7$ & $9.4,7.8-10.9$ \\
\hline $\begin{array}{l}\text { Densidad vascular interna } \\
6 \times 6 \mathrm{~mm}\end{array}$ & $17.1-19.8$ & $18.4,19.0-19.3$ \\
\hline $\begin{array}{l}\text { Densidad vascular externa } \\
6 \times 6 \mathrm{~mm}\end{array}$ & $17.5-20.1$ & $19.3,18.8-19.6$ \\
\hline $\begin{array}{l}\text { Densidad vascular competa } \\
6 \times 6 \mathrm{~mm}\end{array}$ & $17.2-19.7$ & $18.9,18.4-19.2$ \\
\hline $\begin{array}{l}\text { Área de la zona avascular foveal } \\
3 \times 3 \mathrm{~mm}\end{array}$ & $0.13-0.48$ & $0.32,0.24-0.36$ \\
\hline $\begin{array}{l}\text { Área de la zona avascular foveal } \\
6 \times 6 \mathrm{~mm}\end{array}$ & $0.13-0.44$ & $0.29,0.24-0.35$ \\
\hline $\begin{array}{l}\text { Densidad de perfusión central } \\
3 \times 3 \mathrm{~mm}\end{array}$ & $0.10-0.27$ & $0.18,0.15-0.21$ \\
\hline $\begin{array}{l}\text { Densidad de perfusión interna } \\
3 \times 3 \mathrm{~mm}\end{array}$ & $0.35-0.44$ & $0.38,0.37-0.39$ \\
\hline $\begin{array}{l}\text { Densidad de perfusión completa } \\
3 \times 3 \mathrm{~mm}\end{array}$ & $0.32-0.41$ & $0.38,0.37-0.39$ \\
\hline $\begin{array}{l}\text { Densidad de perfusión central } \\
6 \times 6 \mathrm{~mm}\end{array}$ & $0.11-0.34$ & $0.21,0.18-0.25$ \\
\hline $\begin{array}{l}\text { Densidad de perfusión interna } \\
6 \times 6 \mathrm{~mm}\end{array}$ & $0.40-0.48$ & $0.45,0.44-0.46$ \\
\hline $\begin{array}{l}\text { Densidad de perfusión externa } \\
6 \times 6 \mathrm{~mm}\end{array}$ & $0.43-0.50$ & $0.48,0.47-0.49$ \\
\hline $\begin{array}{l}\text { Densidad de perfusión completa } \\
6 \times 6 \mathrm{~mm}\end{array}$ & $0.42-0.48$ & $0.47,0.46-0.47$ \\
\hline
\end{tabular}

Tabla 2. Comparación de las medianas de las variables correspondientes entre protocolos

\begin{tabular}{|c|c|c|c|}
\hline Variable & $\begin{array}{c}3 \times 3 \mathrm{~mm} \\
\text { (mediana, rango } \\
\text { intercuartílico) }\end{array}$ & $\begin{array}{c}6 \times 6 \mathrm{~mm} \\
\text { (mediana, rango } \\
\text { intercuartílico) }\end{array}$ & $p^{*}$ \\
\hline $\begin{array}{l}\text { Densidad vascular } \\
\text { central }\left(\mathrm{mm}^{-1}\right)\end{array}$ & $10.3,8.57-12.02$ & $9.40,7.8-10.95$ & $<0.001$ \\
\hline $\begin{array}{l}\text { Área de la zona } \\
\text { avascular foveal } \\
\left(\mathrm{mm}^{-2}\right)\end{array}$ & $0.32,0.24-0.36$ & $0.29,0.24-0.35$ & $<0.001$ \\
\hline $\begin{array}{l}\text { Densidad de } \\
\text { perfusión central } \\
(\%)\end{array}$ & $0.18,0.15-0.21$ & $0.21,0.18-0.25$ & $<0.001$ \\
\hline
\end{tabular}

Tabla 3. Correlaciones entre variables

Variables

Rho de $\quad$ p Spearman

Densidad vascular central $3 \times 3$ mm/área $3 \times 3 \mathrm{~mm}$

$-0.87<0.00$

Densidad vascular central $3 \times 3 \mathrm{~mm} /$ densidad de perfusión central $3 \times 3 \mathrm{~mm}$

Densidad vascular interna $3 \times 3 \mathrm{~mm} /$ densidad vascular completa $3 \times 3 \mathrm{~mm}$

Densidad vascular interna $3 \times 3 \mathrm{~mm} /$ densidad de perfusión interna $3 \times 3 \mathrm{~mm}$

Densidad vascular completa $3 \times 3 \mathrm{~mm} /$ densidad de perfusión completa $3 \times 3 \mathrm{~mm}$

Área de la zona avascular foveal $3 \times 3 \mathrm{~mm} /$ densidad de perfusión central $3 \times 3 \mathrm{~mm}$

Densidad de perfusión interna $3 \times 3 \mathrm{~mm} /$ densidad de perfusión completa $3 \times 3 \mathrm{~mm}$

Densidad vascular central $6 \times 6 \mathrm{~mm} / a ́ r e a$ $6 \times 6 \mathrm{~mm}$

Densidad vascular central $6 \times 6 \mathrm{~mm} /$ densidad de perfusión central $6 \times 6 \mathrm{~mm}$

Densidad de perfusión interna $6 \times 6 \mathrm{~mm} /$ densidad de perfusión completa $6 \times 6 \mathrm{~mm}$

Densidad vascular interna $6 \times 6 \mathrm{~mm} /$ densidad de perfusión interna $6 \times 6 \mathrm{~mm}$

Densidad vascular e $\times$ terna $6 \times 6 \mathrm{~mm} /$ densidad vascular completa $6 \times 6 \mathrm{~mm}$

Densidad vascular completa $6 \times 6 \mathrm{~mm} /$ densidad de perfusión e x terna $6 \times 6 \mathrm{~mm}$

Densidad vascular complete $6 \times 6 \mathrm{~mm} /$ densidad de perfusión completa $6 \times 6 \mathrm{~mm}$

Área de la zona avascular foveal $6 \times 6 \mathrm{~mm} /$ densidad de perfusión central $6 \times 6 \mathrm{~mm}$

Densidad de perfusión interna $6 \times 6 \mathrm{~mm} /$ densidad de perfusión completa $6 \times 6 \mathrm{~mm}$

Densidad de perfusión e x terna $6 \times 6 \mathrm{~mm} /$ densidad de perfusión completa $6 \times 6 \mathrm{~mm}$

Densidad vascular central $3 \times 3 \mathrm{~mm} /$ densidad de perfusión central $6 \times 6 \mathrm{~mm}$

Densidad vascular central $6 \times 6 \mathrm{~mm} /$ densidad de perfusión central $3 \times 3 \mathrm{~mm}$

\begin{tabular}{|l|l|}
\hline 0.98 & $<0.001$ \\
\hline 0.96 & $<0.001$ \\
\hline 0.88 & $<0.001$ \\
\hline 0.9 & $<0.001$ \\
\hline 0.84 & $<0.001$ \\
\hline 0.96 & $<0.001$ \\
\hline 0.84 & $<0.001$ \\
\hline 0.99 & $<0.001$ \\
\hline 0.83 & $<0.001$ \\
\hline 0.86 & $<0.001$ \\
\hline 0.85 & $<0.001$ \\
\hline 0.001 & $<0.001$ \\
\hline & $<001$ \\
\hline 0.001
\end{tabular}

variables fueron distintos entre los protocolos, lo que hace imprecisa la comparación entre ellos

Hubo mejores correlaciones entre las densidades vascular y de perfusión dentro de un protocolo que entre los valores de densidad vascular y de perfusión de distintos protocolos (p. ej., densidad vascular 

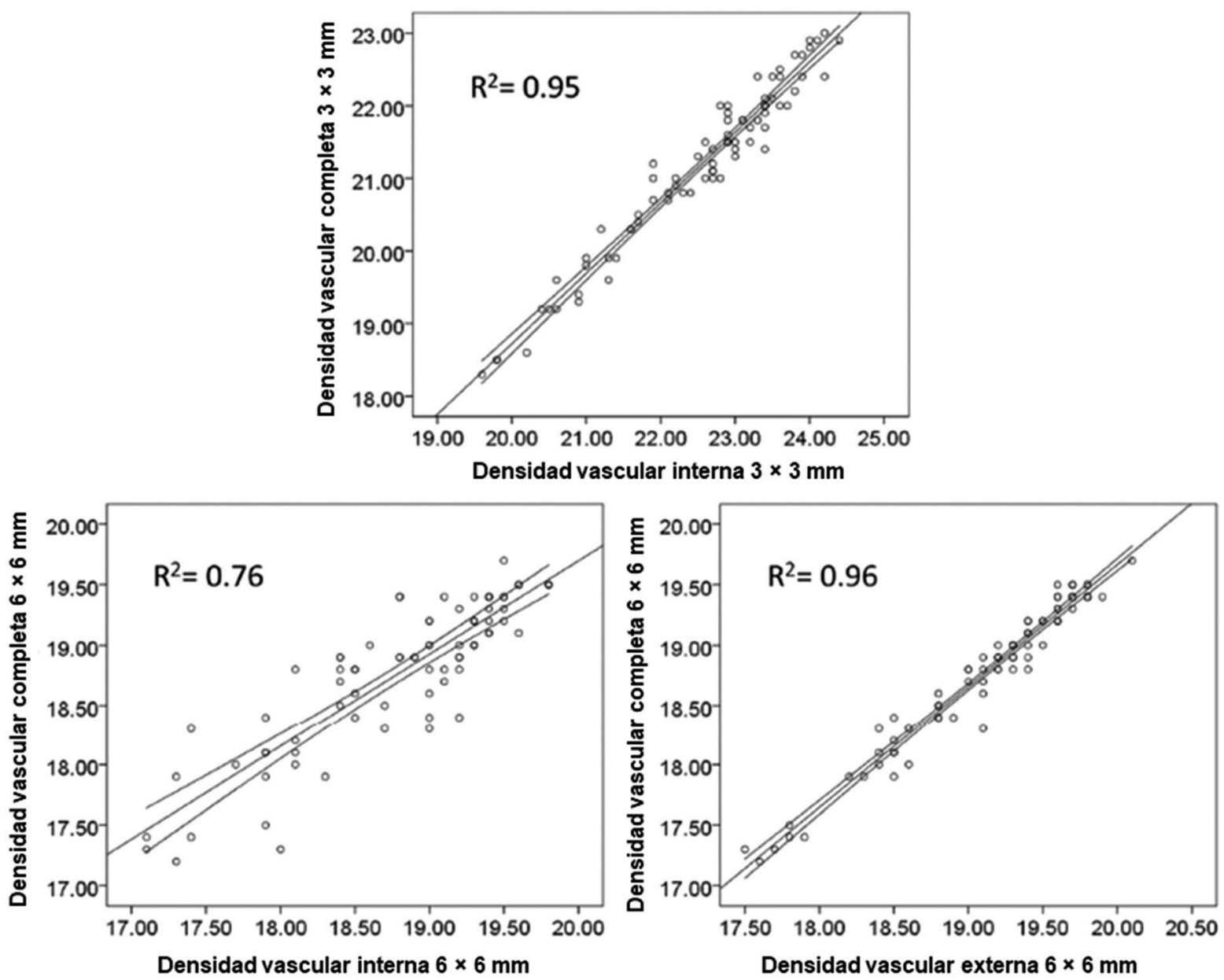

Figura 1. Diagrama de dispersión entre las densidades vasculares interna y completa en el protocolo de $3 \times 3 \mathrm{~mm}$, y entre las densidades vasculares interna y externa y la densidad vascular completa en el protocolo de $6 \times 6 \mathrm{~mm}$. El coeficiente de determinación fue alto entre la densidad vascular interna y la densidad de vascular completa en el protocolo de $3 \times 3 \mathrm{~mm}$, y disminuyó entre la densidad vascular interna y la densidad vascular completa en el protocolo de $6 \times 6 \mathrm{~mm}$. La R2 entre la densidad vascular externa y la densidad vascular completa en el protocolo de 6 $x 6 \mathrm{~mm}$ fue mayor que la existente entre las densidades vasculares interna y completa en el protocolo de $3 \times 3 \mathrm{~mm}$; el protocolo de $3 \times 3 \mathrm{~mm}$ mide la región interna, mientras que el de $6 \times 6 \mathrm{~mm}$ mide principalmente la región externa.

central en el protocolo de $3 \times 3 \mathrm{~mm}$ y densidad de perfusión central en el protocolo de $6 \times 6 \mathrm{~mm}$ ); este patrón requeriría mantener constante un protocolo para comparaciones longitudinales, aun cuando distintas patologías podrían necesitar la evaluación de un área específica.

Ho et al. compararon los hallazgos en ojos con retinopatía diabética no proliferativa entre protocolos de $3 \times 3 \mathrm{~mm}$ y de $6 \times 6 \mathrm{~mm}$ con un equipo distinto de angiografía por tomografía de coherencia óptica y concluyeron que el protocolo de $3 \times 3 \mathrm{~mm}$ era mejor para evaluar la zona avascular foveal; encontraron una proporción mayor de microaneurismas en el protocolo de $6 \times 6 \mathrm{~mm}$. También concluyeron que las diferencias eran el resultado de una mayor densidad de rastreo en el protocolo de $3 \times 3 \mathrm{~mm}$ y de que el protocolo de $6 \times 6 \mathrm{~mm}$ evaluaba una superficie mayor..$^{12} \mathrm{El}$ equipo que usaron tiene rastreos constantes de $304 \times 304$ tanto para el protocolo de $3 \times 3 \mathrm{~mm}$ como para el de $6 \times 6 \mathrm{~mm}$, mientras que el Angioplex realiza $245 \times 245$ rastreos para el protocolo de 3 x $3 \mathrm{~mm}$ y 350 x 350 rastreos para el de $6 \times 6 \mathrm{~mm} .^{6}$

Dalan et al. reportaron una consistencia excelente entre los protocolos de $3 \times 3 \mathrm{~mm}$ y de $6 \times 6 \mathrm{~mm}$ para las densidades central e interna vascular (coeficiente de correlación intraclase: 0.89) y de perfusión (coeficiente de correlación intraclase: 0.80 ), en ojos normales evaluados con el Angioplex..$^{13}$ En nuestro estudio se encontró que esas mediciones difirieron estadísticamente entre ambos protocolos; por lo tanto, aunque 

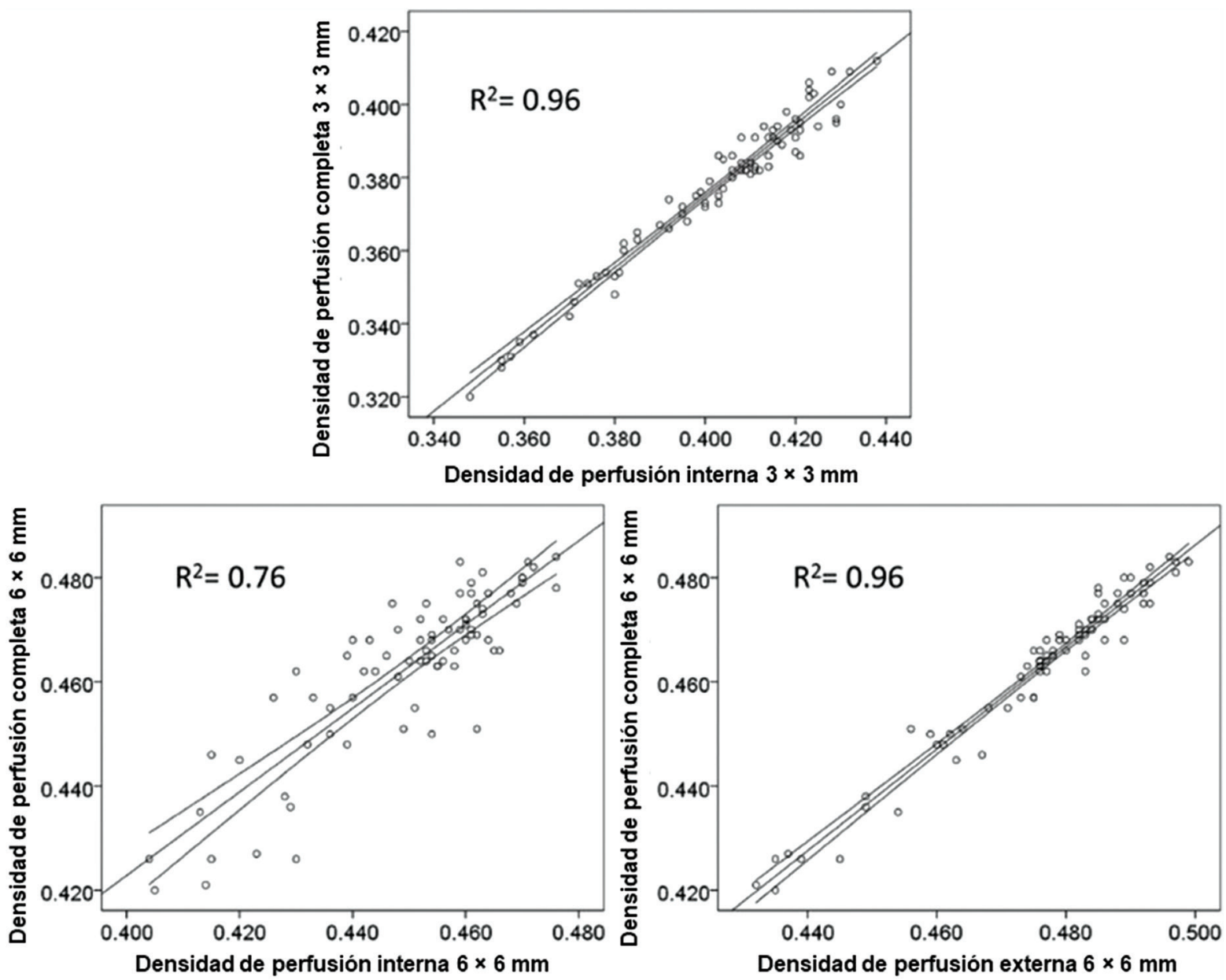

Figura 2. Diagrama de dispersión entre las densidades de perfusión interna y completa en el protocolo de $3 \times 3 \mathrm{~mm}$, y entre las densidades de perfusión interna y externa y la densidad de perfusión completa en el protocolo de $6 \times 6 \mathrm{~mm}$. Al igual que en las mediciones de densidad vascular, el protocolo de $3 \times 3 \mathrm{~mm}$ mide la región interna, y el de $6 \times 6 \mathrm{~mm}$ mide preferentemente la región externa. La $R^{2}$ entre la densidad de perfusión completa y la densidad de perfusión completa es mayor que entre la densidad de perfusión interna y la densidad de perfusión completa, en el protocolo de $6 \times 6 \mathrm{~mm}$.

tengan un buen coeficiente de correlación intraclase, el valor de una misma medición obtenida con diferentes protocolos fue mayor para la densidad vascular en el protocolo de $3 \times 3 \mathrm{~mm}$, y más alta para la densidad de perfusión en el protocolo de $6 \times 6 \mathrm{~mm}$.

Xiao et al. reportaron diferencias semejantes: concluyeron que había una buena reproducibilidad entre los protocolos de $3 \times 3 \mathrm{~mm}$ y de $6 \times 6 \mathrm{~mm}$ del Angioplex $y$ advirtieron que los valores obtenidos por ambos protocolos no podían compararse directamente. ${ }^{14}$ Dalan et al. atribuyeron la diferencia a la distancia promedio de $12.2 \mu \mathrm{m}$ entre rastreos en el protocolo de $3 \times 3 \mathrm{~mm}$, comparada con el promedio de $17.1 \mu \mathrm{m}$ entre rastreos en el protocolo de $6 \times 6 \mathrm{~mm}^{13}$ Sin embargo, los coeficientes de determinación que encontramos muestran que las densidades vascular y de perfusión completas se explican mejor por las densidades externas en el protocolo de $6 \times 6 \mathrm{~mm}$ que por las densidades internas. Entonces, el protocolo de 6 x $6 \mathrm{~mm}$ se enfoca en la región externa del rastreo, lo cual podría explicar que su evaluación de la densidad de la densidad vascular en sus $3 \mathrm{~mm}$ centrales sea menos extensa que en el protocolo de $3 \times 3 \mathrm{~mm}$.

Estos hallazgos no explican por qué hubo medianas más altas de las densidades de perfusión en el protocolo de $6 \times 6 \mathrm{~mm}$, lo cual también fue reportado por Dong con otro equipo de angiografía por tomografía de coherencia óptica. ${ }^{15}$ En nuestra muestra la distribución de las variables de perfusión fue normal en el protocolo de $3 \times 3 \mathrm{~mm}$, pero no en el de $6 \times 6 \mathrm{~mm}$; aunque la muestra fue mayor que otras que han reportado diferencias en los valores de densidad de 
perfusión, todavía se requiere determinar si los valores de las densidades de perfusión son todavía mayores en el protocolo de $6 \times 6 \mathrm{~mm}$ en una muestra con distribución normal.

Era esperado encontrar un coeficiente de determinación alto entre las densidades vascular y de perfusión dentro de un protocolo, pero se desconocían los valores entre protocolos, lo cual es una pregunta común cuando el paciente solo tiene un reporte de densidad vascular, con reportes de distintos protocolos. Los coeficientes de determinación entre densidades vascular y de perfusión entre los distintos protocolos fueron menores a $\mathrm{R}^{2}=0.7$.

Los protocolos de rastreo se diseñan para propósitos distintos, y es de esperarse que cambien las regiones que evalúen; sin embargo, algunos aumentan el área de rastreo sin cambiar las mediciones originales y otros, como el Angioplex, cambian el énfasis de la evaluación hacia la región nueva explorada, lo que modifica las mediciones de las zonas que se evalúan en común, En el primer caso los valores de las mediciones comunes podrían intercambiarse, mientras que en el segundo no se podría; el protocolo de $6 \times 6 \mathrm{~mm}$ está diseñado para evaluar la perifóvea, y la fóvea y la parafóvea se analizan mejor con el protocolo de $3 \times 3 \mathrm{~mm}$.

Con respecto al área de la zona avascular foveal, fue mayor en el protocolo de $3 \times 3 \mathrm{~mm}$, probablemente por los distintos patrones topográficos de rastreo. Los coeficientes de determinación entre esta variable y las de densidad vascular y perfusión fueron cercanos a $R^{2}=0.7$. La dimensión de la zona avascular foveal es una referencia habitual en la angiografía por tomografía de coherencia óptica tanto en ojos con enfermedades ${ }^{16}$ como en ojos sin ellas; 4,17 los valores del área de la zona avascular foveal fueron mayores en el protocolo de $3 \times 3 \mathrm{~mm}$, lo cual limitaría la comparación directa con un protocolo de $6 \times 6 \mathrm{~mm}$.

Una fortaleza del estudio fue la evaluación de densidades vascular y de perfusión tanto regionales como completa, lo que permitió detectar las distintas correlaciones dentro de un protocolo de rastreo. Una potencial debilidad fue que la edad de los sujetos es menor a la reportada por otros estudios, que suelen seleccionar muestras que tengan edad semejante a la de los pacientes con enfermedades retinianas que evalúan. Consideramos que evaluar sujetos jóvenes reduciría la probabilidad de encontrar cambios retinianos causados por enfermedades vasculares no diagnosticadas, y que esta información podría ser útil para comparaciones futuras con otros grupos de edad.

\section{Conclusiones}

Las densidades vasculares y de perfusión en la mácula de sujetos sanos tienen una correlación alta dentro de los protocolos de $3 \times 3 \mathrm{~mm}$ y de $6 \times 6 \mathrm{~mm}$, que es mejor que las correlaciones entre ambas variables y el área de la zona avascular foveal. Las mediciones de los protocolos de $3 \times 3 \mathrm{~mm}$ y de $6 \times 6 \mathrm{~mm}$ no deben intercambiarse, porque cada uno mide preferentemente una región distinta de la mácula.

\section{Conflicto de intereses}

Los autores declaran que no existe conflicto de interés financiero ni de algún otro tipo en relación con el trabajo descrito.

\section{Financiamiento}

El estudio solo recibió financiamiento de la Clínica de Microcirugía Ocular Avanzada Dr. Enrique Monares García.

\section{Responsabilidades éticas}

Protección de sujetos humanos y animales. Los autores declaran que no se realizaron experimentos en humanos o animales para este estudio.

Confidencialidad de los datos. Los autores declaran haber seguido los protocolos de su centro de trabajo sobre la publicación de datos de pacientes.

Derecho a la privacidad y consentimiento informado. Los autores declaran que en este artículo no aparecen datos de pacientes.

\section{Bibliografía}

1. Spaide RF, Fujimoto JG, Waheed NK, Sadda SR, Staurenghi G. Optical coherence tomography angiography. Progr Retin Eye Res. 2018;64:1-55.

2. Kashani AH, Chen CL, Gahm JK, Zheng F, Richter GM, Rosenfeld PJ. Optical coherence tomography angiography: a comprehensive review of current methods and clinical applications. Progr Retin Eye Res. 2017;60:66-100.

3. Zhao Q, Yang WL, Wang XN, Wang RK, You QS, Chu ZD. Repeatability and reproducibility of quantitative assessment of the retina microvasculature using optical coherence tomography angiography based on optical microangiography. Biomed Environ Sci. 2018;31:407-12.

4. Guo J, She X, Liu X, Sun X. Repeatability and reproducibility of foveal avascular zone area measurements using AngioPlex spectral domain optical coherence tomography angiography in healthy subjects. Ophthalmologica. 2017:237:21-8.

5. Lee TH, Lim HY, Nam KY, Kim K, Kim JY. Factors affecting repeatability of assessment of the retinal microvasculature using optical coherence tomography angiography in healthy subjects. Sci Rep. 2019;9:1691.

6. Rosenfeld PJ, Durbin MK, Rosiman L, Zheng F, Miller A, Robbins G. ZEISS angioplex spectral domain optical coherence tomography angiography: technical aspects. Dev Ophthalmol. 2016;56:18-29.

7. Shiijara H, Sakamoto T, Yamashita T, Kakiuchi N, Otsuka H, Terasaki H. Reproducibility and differences in area of foveal avascular zone measured by three different optical coherence tomographic angiography instruments. Sci Rep. 2017;7:9853. 
8. Dave PA, Dansigani KK, Jabeen A, Jabeen A, Hasnat AM, Vupparaboina KK. Comparative evaluation of foveal avascular zone on two optical coherence tomography angiography devices. Optom Vis Sci. 2018;95:602-7.

9. Chung CS, Nesper PL, Park JJ, Fawzi AA. Comparison of Zeiss Cirrus and Optovue RTvue OCT angiography Systems: a quantitative and qualitative approach examining the three capillary networks in diabetic retinopathy. Ophthalmic Surg Lasers Imaging Retina. 2018:49:e198-e205.

10. Hussain N, Hussain A. Diametric measurement of foveal avascular zone in healthy young adults using optical coherence tomography angiography. Int J Retin Vitr. 2016;2:27.

11. Corvi F, Pellegrini M, Erba S, Cozzi M, Staurenghi G, Giani A. Reproducibility of vessel density, fractal dimension, and foveal avascular zone using 7 different optical coherence tomography angiography devices. Am J Ophthalmol. 2018;186:25-31.

12. Ho J, Dans K, You Q, Nudlleman ED, Freeman WR. Comparison of 3 $\mathrm{mm} \times 3 \mathrm{~mm}$ versus $6 \times 6 \mathrm{~mm}$ optical coherence tomography angiography scan sizes in the evaluation of non-proliferative diabetic retinopathy. Retina. 2019;39:259-64.
13. Dalan D, Nandini, P, Angayarkanni N, Kaviarasan K, Thanikachalam S Das UN. Interchangeability of retinal perfusion indices in different-sized angiocubes: an optical coherence tomography angiography study in diabetic retinopathy. Indian J Ophthalmol. 2020;68:484-9.

14. Xiao H, Liu X, Liao L, Tan K, Ling Y, Zhong Y. Reproducibility of foveal avascular zone and superficial macular retinal vasculature measurements in healthy eyes determined by two different scanning protocols of optical coherence tomography angiography. Ophthalmic Res. 2019;16:1-8.

15. Dong J, Jia YD, Zhang S, Jia Y, Huang D. Interchangeability and reliability of macular perfusion parameter measurements using optical coherence tomography angiography. Br J Ophthalmol. 2017;101:1542-9.

16. Lu Y, Wang JC, Zeng R, Katz R, Vavvas DG, Miller JW, et al. Quantitative comparison of microvascular metrics on three optical coherence tomography angiography devices in chorioretinal disease. Clin Ophthalmol. 2019:13:2063-9.

17. Flavarjani KG, Shenazandi H, Naseri D, Anvari P, Kazemi P, Aghamohammadi F. Foveal avascular zone and vessel density in healthy subjects: an optical coherence tomography angiography study. J Ophthalmic Vis Res. 2018;13:260-5. 